\title{
SUPPRESSING EPIDEMIC SPREADING VIA DIFFERENTIAL SUSCEPTIBILITY MODELS
}

\author{
Mehdi Afshar ${ }^{1 *}$ \\ *1 Department of Mathematics and Statistics, Faculty of Basic Sciences,Zanjan Branch, Islamic \\ Azad University, Zanjan, IRANEmail:mafshar@iauz.ac.ir
}

*Corresponding Author: -

\begin{abstract}
: -
This paper considers the optimal control problem for some models using differential equations in epidemiology. In particular, we consider the differential susceptibility model (DS). Because of apparent diversity of examples, the idea of dividing susceptible population into two subgroups examined. For example, in plenty of diseases, disease processes is different in male or female, children or adult, addicted or non-addicted, and so on. We show that it would be useful to consider this difference to reduce the costsin epidemic spreading. We want to exert some parameters in differential susceptibility model to control the epidemic spreading. The optimal control represents a drug treatment and Prevention strategies. Existence and uniqueness results for the optimal control are discussed. Finally, numerical examples are given for illustration.
\end{abstract}

Keywords: Mathematical modelling, Epidemiological model, Differential susceptibility, Optimal Control.

Mathematics Subject Classification: 93A30, 49J15, 92D30 


\section{INTRODUCTION}

Optimal control theory is a branch of mathematics that can be used in various biological models [Lenhart 2007]. For instance,this tool is useful in epidemiological models. In [Gaff et . 2009], optimal control is used in restraining the spread of infectious diseases. They try to find the efficient control strategy to minimize the number of individuals who were infected in epidemic spreading. For this purpose, they used treatment and vaccination as control measures.

There are more specific examples of this kind of works. In [Jung et al. 2002], optimal control theory is applied to modeling a two-strain tuberculosis model or or applied to investigate optimal strategies for controlling the spread of malaria [Folashade et al. 2012]. The study by [Kirschner et al. 1997] used optimal control theory to establish the optimal treatment strategy to manage the antiretroviral drug in individuals who were HIV positive.GJ Abiodun et al investigated the HIV/AIDS epidemic in a population which experiences a significant flow of immigrants. They introduced and analyzed a mathematical model that describes the dynamics of HIV infectionamong the immigrant youths and how parental care can reduce or prevent the spread of the disease in the population [abiodun et al.2013].[Fister and Donnelly 2005] also used optimal control theory to state the elimination condition of tumor cells in individuals who were under treatment for Cancer.

The structure of a population or group which is socially comminuted plays an important role in the dynamics ofa disease transmission [Castillo- Chavez et al. 1989]. Epidemiological models are almost multigroup models. Groups can be classified into geographical groups such as cities, countries and communities, behavioral groups like different patterns of contact and high risk groups or epidemiological groups like vertical transmission and co-infection of multiple origins of the disease agent [Thieme 2003].

A well-known example for these subpopulations is the core group, subgroups which are sexually active extensively [Hethcote and York 1984]. It is clear that the transmission and interaction within and among core subgroups is important in the transmission of HIV/AIDS[Huang et al.1992]. Medical evidences represent that itis well-advised to introduce differential susceptibility (DS) as well [Thieme 2003] and [Hyman et al.2001]. Genetic variation of susceptible individuals and the active effect of vaccination cause to the differentiation of susceptibility on infection. In some diseases, vaccinated individuals may still get the disease, but it varies between the individuals. The importance of differential susceptibility has been studied in the immune system too [Cleary 1988], [Horikoshi et al.2001] and [Waterai et al. 2001]. Differential susceptibility of infection can

occur after vaccination is administered for infectious diseases. Prevention of disease is the most efficient way of handling rubeola. Despite the extensive vaccination programs, many women remain susceptible such that two doses of liveattenuated vaccine should be administered for prevention [Signore 2001].

Hyman et al, studied on differential susceptibility models and their impacts on disease transmission. They investigated on rubeola, HB and HIV based on DS model [Hyman and Li 2005]. Effect of variation in susceptibility to measles, smallpox and wooping cough epidemics have been studied in [Duncan et al.1996] and [Bolker and Grenfell 1993]. Rigorous study on basic reproduction number, local analysis and global analysis of DS model has been presented in [Hyman and $\mathrm{Li}$ 2005].

We develop optimal control formulation of DS model with two groups of susceptible individuals. The modeling process and optimal control model are presented in the next section. The basic reproduction ratio will be computed in section 3 . We study the existence and uniqueness of optimal control results in section 4 . The optimal control is derived in terms of the adjoints and states by Pontryagin Maximum Principle (PMP) in section 5. The control strategies are considered. Uniqueness of optimal control problem is proven in section 6 and finally the results of these studies are illustrated with numerical examples.

\section{THEORETICAL MODEL}

Consider the epidemic spreading in a homogeneous population that approaches a steady state $\mathrm{S}^{0}$, if there is no disease infection. We assume that infected individuals become immune or are removed from the susceptible population after they escaped from the infection. The dynamics of the transmission is modeled with an SIR(susceptible-infected recovered) model. Here, we divide the susceptible into two subgroups, $S_{1}$ and $S_{2}$ and suppose that the individual in each group have different susceptibility and have homogeneous susceptibility based on their inherent susceptibility, in such way that the input flow into group $\mathrm{S}_{\mathrm{i}}$ is $\mu \mathrm{p}_{\mathrm{i}} \mathrm{S}^{0}$ with $p_{1}+p_{2}=1$. The susceptible individuals stay in those groups until they become infected. We assume full

immunity of recovered individuals such that these individuals are no longer susceptible after they recovered,or complete isolations after individuals are infected and diagnosed. The model is illustrated in Figure(1) and transmission dynamics of infection are governed by the system of differential equations below [Hyman and Li 2005].

$$
\begin{aligned}
& 1 \geqslant \mu p_{1} S^{0}-\mu S_{1}-\lambda_{1} S_{1} I \\
& 2 \mu p_{2} S^{0}-\mu S_{2}-\lambda_{2} S_{2} I \\
& \lambda_{1} S_{1} I+\lambda_{2} S_{2} I-(\mu+v+\delta) I \\
& 2 v I-(\xi+\mu) R
\end{aligned}
$$

Where $\mathrm{S}_{1}, \mathrm{~S}_{2}$, I , and $\mathrm{R}$ denote the susceptible in the first subgroup, susceptible in the second subgroups,infectives and recovered or removed individuals. 


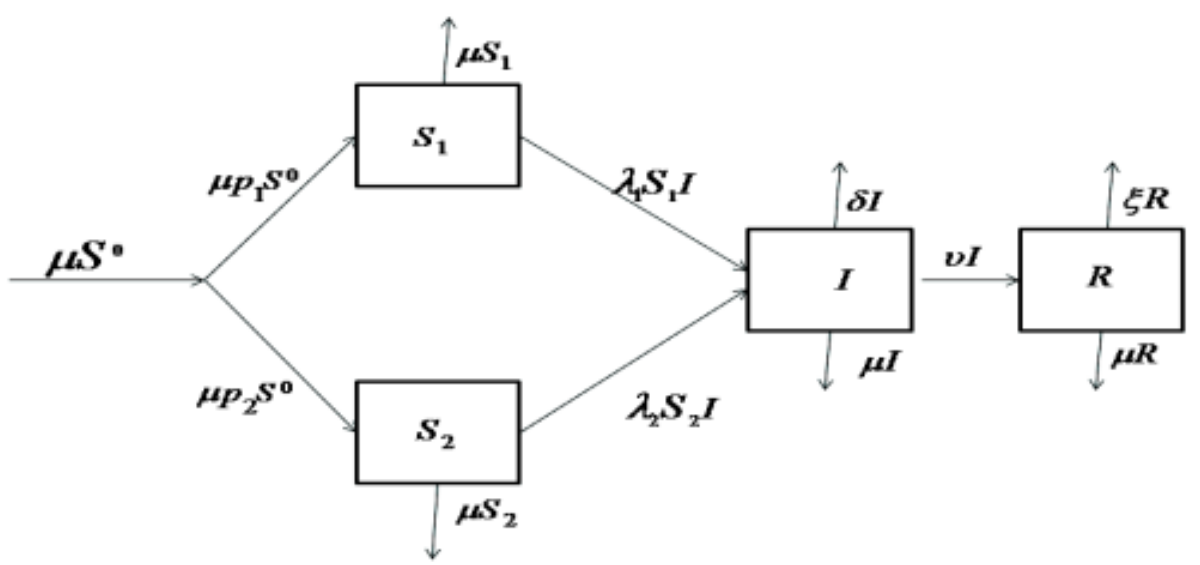

The following parameters appear in our model:

Figure 1: Model

$p i$ : per capita input flow rate entering $S i$;

$\mu$ : natural death rate;

$v:$ the rate at which infectives are removed or become immune;

$\delta$ : disease-induced mortality rates for the infectives;

$\xi$ : disease-induced mortality rates for the removed individuals;

$\lambda i$ : the rate of infection for each infective groups;

The infectivity rate $\lambda i$ is given by $\lambda_{i}=\beta c \alpha_{i}, \mathrm{i}=1,2$ where $\alpha_{\mathrm{i}}$ is the susceptibility of susceptible individuals in $\mathrm{S}_{\mathrm{i}}, \beta$ is the infectious rate of infected individuals, $c=c(N)$ is the average number of contacts per individual, with $N=S_{1}+S_{2}+I$ $+R$, the total population size. Using the same parameters and class names as in the model (1), we suggested the following ODEs system (2) describing the model with controls.

$$
\begin{aligned}
& 2 p_{1} S^{0}-\mu S_{1}-\lambda_{1} S_{1} I\left(1-u_{1}\right) \\
& 2 \geqslant p_{2} S^{0}-\mu S_{2}-\lambda_{2} S_{2} I\left(1-u_{2}\right) \\
& \lambda_{1} S_{1} I\left(1-u_{1}\right)+\lambda_{2} S_{2} I\left(1-u_{2}\right)-\left(\mu+v+\delta+u_{3}\right) I \\
& \left(v+u_{3}\right) I-(\xi+\mu) R
\end{aligned}
$$

The control functions $u_{1}(\mathrm{t}), u_{2}(\mathrm{t})$ and $u_{3}(\mathrm{t})$ have to be bounded on $[0,1]$ and Lebesgue integrable functions. $\mathrm{u}_{1}(\mathrm{t})$ and $\mathrm{u}_{2}(\mathrm{t}$ ) measure the time dependent efforts on the preventive strategy of susceptible individuals in $S_{1}$ and $S_{2}$ respectively, to reduce the number of individuals that may be infectious. The control function $u_{3}(\mathrm{t})$ measures the time dependent efforts on the treatment of infected individuals. This control will have an impacton the output flow of people from the objective functional to be minimized is:

$$
J\left(u_{1}, u_{2}, u_{3}\right)=\overbrace{0}^{T}(I, U) d t=\underbrace{T}_{0} I^{2}+B u_{1}{ }^{2}+C u_{2}{ }^{2}+D u_{3}{ }^{2} d t
$$

Here, A, B ,C and D are adjustment parameters. They are converting the dimension from population number into cost expended over a finite time period of $\mathrm{T}$ years. The first term, $A I^{2}$ is the cost of death due to disease and the remaining expressions $B u_{1}^{2}+C u_{2}^{2}+D u_{3}^{2}$ are costs for implementation of the three controls.

We seek an optimal control triple $\left(\mathrm{u}_{1}{ }^{*}, \mathrm{u}_{2} *, \mathrm{u}_{3} *\right)$ such that

$$
J\left(u_{1} *, u_{2} *, u_{3} *\right)=\min \left\{J\left(u_{1}, u_{2}, u_{3}\right) \mid\left(u_{1}, u_{2}, u_{3}\right) \nabla U\right\}
$$

Where $\left.\left.U=\left\{\left(u_{1}, u_{2}, u_{3}\right) \mid u_{i} \text { measurable, } 0\right\rangle_{u_{i}} \vartheta_{1}, t>0, T\right], i=1,2,3\right\}$ is the control set.

There are important points about objective functional adjustment. For instance, when one emphasizes onnumber of deaths from the disease, we can change the objective functional to

$$
J\left(u_{1}, u_{2}, u_{3}\right)=\underbrace{T}_{0}(I, U) d t=\underbrace{T}_{0} \boldsymbol{T}+B u_{1}{ }^{2}+C u_{2}{ }^{2}+D u_{3}{ }^{2} d t
$$

Here, $I_{1}, I_{2}>[0,1]$ and $I_{i}{ }^{2}<I_{i}$ for $\mathrm{i}=1,2$ and consequently the optimal control focus on $I_{i}$ for $i=1,2$. We illustrate these points in numerical examples. 


\section{BASIC REPRODUCTION NUMBER}

The global behavior of the DS model depends on the basic reproduction number, i.e., an average number ofsecondary cases produced by a single infective individual, who is introduced into an entirely susceptible population. System (1) has an infection-free equilibrium in which the component of infective equals to zero and other susceptible components are positive. According to its definition, $R_{0}$ is a multiplicative coefficient.

This suggests that for $R_{0}>1$, there will be an increase in cases leading to an epidemic, and the disease will disappear if $R_{0}<1$. Denote this infection-free equilibrium by $E=\left(p_{1} S^{0}, p_{2} S^{0}, 0,0\right)$. Analyzing the local stabilityof this point gives the epidemic threshold condition, $R_{0}$. E is locally asymptotically stable if $R_{0}<1$, and unstable if $R_{0}<1$.

$$
R_{0}=\frac{\eta \beta S^{0}}{\mu+v}\left(\alpha_{1} p_{1}+\alpha_{2} p_{2}\right)=\frac{\beta c S^{0}}{\mu+v}\left(\alpha_{1} p_{1}+\alpha_{2} p_{2}\right)=\frac{r \beta}{\mu+v}\left(\alpha_{1} p_{1}+\alpha_{2} p_{2}\right) \text { (4). }
$$

at which $\eta=\frac{c\left(S^{0}\right)}{S^{0}}$. Note that the mean number of contacts is $c\left(S^{0}\right)=r$ the mean duration of infection for this model is $\tau:=\frac{1}{\mu+v}$, and the mean infectivity for each group is $\bar{\beta}_{i}:=\beta \alpha_{i}$. We investigate the effect of different prevention strategies on the spread of infectious diseases within a population. The majority of existing papersfall into one of two groups. In the first group, prevention strategies are modeled by a constant parameter and the goal is to understand how changing the value of the parameter changes the dynamics of the system.

Often the aim is to determine the best parameter value for a given performance measure. In this manner wecan compute basic reproductive ratio,

$$
R_{0}=\frac{r \beta}{\mu+v+u_{3}}\left(\alpha_{1} p_{1}\left(1-u_{1}\right)+\alpha_{2} p_{2}\left(1-u_{2}\right)\right)
$$

In the second group, prevention strategies are allowed to vary as a function of time and the goal is to determine the best function for a given performance measure. We will investigate this point of view in thefollowing.

\section{DERIVING OPTIMAL SOLUTION}

In this section, we derive the optimal control triple system for minimizing the functional 3 subject to (1). Inorder to derive the necessary conditions for this optimal control, we use Pontryagin's Maximum Principle [Sethi 2000]. The Hamiltonian is defined as follows:

$$
\begin{aligned}
& H=A I^{2}+B u_{1}^{2}+C u_{2}^{2}+D u_{3}^{2}+ \\
& Y_{1}\left(\mu p_{1} S^{0}-\mu S_{1}-\lambda_{1} S_{1} I\left(1-u_{1}\right)\right)+ \\
& Y_{2}\left(\mu p_{2} S^{0}-\mu S_{2}-\lambda_{2} S_{2} I\left(1-u_{2}\right)\right)+ \\
& Y_{3}\left(\lambda_{1} S_{1} I\left(1-u_{1}\right)+\lambda_{2} S_{2} I\left(1-u_{2}\right)-\left(\mu+v+\delta+u_{3}\right) I\right)+ \\
& Y_{4}\left(\left(v+u_{3}\right) I-(\xi+\mu) R\right)
\end{aligned}
$$

Suppose $\left(u_{1} *, u_{2}^{*}, u_{3}^{*}\right)$ is an optimal control with corresponding states optimal control, the Hamiltonian and adjoint equations are constructed. $\left(S_{1}, S_{2}, I, R\right)$. To characterize the

Theorem 5.1 There exists an optimal control triple $\left(\mathrm{u}_{1} *, \mathrm{u}_{2} *, \mathrm{u}_{3} *\right)$ and corresponding solution $\left(S_{1}, S_{2}, I, R\right)$, that minimizes $J\left(u_{1}, u_{2}, u_{3}\right)$ over $[0, \mathrm{~T}]$. Furthermore, there exists adjoint functions, $\left(Y_{1}, Y_{2}, Y_{3}, Y_{4}\right)$ such that

$$
\begin{aligned}
& 2 Y_{1}\left(\mu+\lambda_{1} I\left(1-u_{1}\right)-Y_{3}\left(\lambda_{1} I\left(1-u_{1}\right)\right)\right. \\
& Y_{4}\left(\mu+\lambda_{2} I\left(1-u_{2}\right)-Y_{3}\left(\lambda_{2} I\left(1-u_{2}\right)\right)\right. \\
& Y_{2}-2 A I+Y_{1}\left(\lambda_{1} S_{1}\left(1-u_{1}\right)\right)+Y_{2}\left(\lambda_{2} S_{2}\left(1-u_{2}\right)\right)+Y_{3}\left(\lambda_{1} S_{1}\left(u_{1}-1\right)+\lambda_{2} S_{2}\left(u_{2}-1\right)+\mu+v+u_{3}\right)
\end{aligned}
$$

with transversality conditions $Y_{i}(T)=0, \quad i=1,2,3,4$. The following characterization holds

$$
\begin{aligned}
& 2 B \\
& 2 B
\end{aligned} *=\min \left\{\max \left\{\frac{\left(Y_{3}-Y_{1}\right)\left(\lambda_{1} S_{1} I\right)}{2 B}, 0\right\}, 1\right\}
$$

PROOF: Applying Pontryagin Maximum Principle, we obtain 


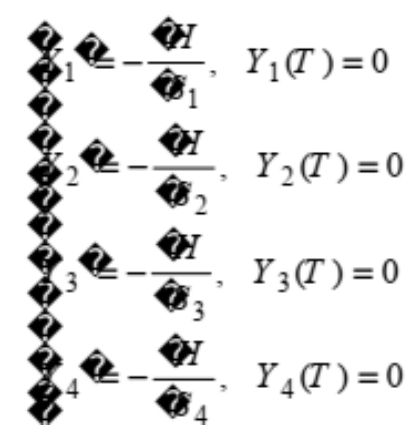

evaluated at the optimal control triple and corresponding states, which results in the stated adjoint system 7 .

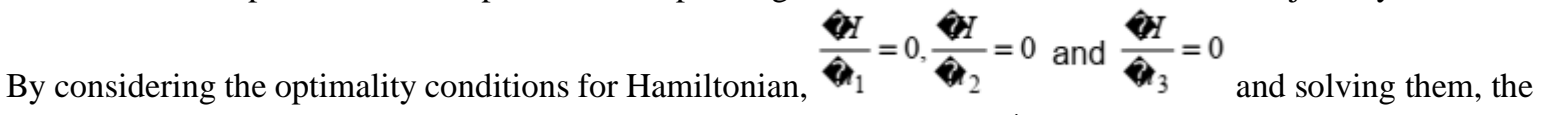
characterization 8 can be derived. To illustrate the characterization of $u_{1} *$ we have

$$
\frac{\partial I}{\lambda_{1}}=2 B u_{1}+\left(Y_{3} \lambda_{1} S_{1} I-Y_{1} \lambda_{1} S_{1} I\right)=0 \vartheta u_{1} *=\frac{\left(Y_{3}-Y_{1}\right) \lambda_{1} S_{1} I}{2 B}
$$

By standard control arguments involving the bounds on the controls, we conclude

$$
u_{1}=2 \begin{array}{ll}
2 B & \\
\text { if } \quad & \frac{\left(Y_{3}-Y_{1}\right) \lambda_{1} S_{1} I}{2 B}<0 \\
2 B & \text { if } \quad \frac{\left(Y_{3}-Y_{1}\right) \lambda_{1} S_{1} I}{2 B}>1
\end{array}
$$

In compact notation, we have $u_{1} *=\min \left\{\max \left\{\frac{\left(Y_{3}-Y_{1}\right) \lambda_{1} S_{1} I}{2 B}, 0\right\}, 1\right\}$. The rest of the control functions $\mathrm{u}_{2}$ and $\mathrm{u}_{3}$ can be found by a similar method.

\section{NUMERICAL RESULTS}

In this section, we study numerically an optimal treatment and prevention strategies of our model. The state system of differential equations and the adjoint system of differential equations together with the control characterization above form the optimal control system to be solved numerically. Since the state equations have initial conditions and the adjoint equations have final time conditions, we cannot solve the optimality system directly by only sweeping forward in time. Thus, an iterative algorithm, forward-backward sweep method (thanks to [Lenhart and Workman 2007]), is used. An initial estimate for the control is made. The statesystem is then solved forward in time from the dynamics using a Runge Kutta method of the fourth order (RK4). Resulting state values are placed in the right hand sides of the adjoint differential equations. Then the adjoint system with given final conditions is solved backward in time, again employing a RK4 method. Both state and adjoint values are used to update the control using the characterization, and then the process is repeated. This iterative process terminates when current state, adjoint, and control values converge sufficiently.

In the following examples, as much as possible, we try to use the justified and estimated parameters of the various examples of the cited references.

\subsection{Example1}

Now we will use the MATLAB program to ascertain how each control parameter affects the solution. This example illustrates how constant parameter control could change the future of epidemic spreading. Let us enter the following values in the model system (1):

\begin{tabular}{|c|c|c|c|}
\hline \multicolumn{4}{|c|}{ Parameters and values } \\
\hline$p_{1}=0.5$ & $r=25$ & $S_{1}(0)=0.5$ & $v=0.25$ \\
\hline$p_{2}=0.5$ & $S^{0}=1$ & $S_{2}(0)=0.45$ & $\beta=0.2$ \\
\hline$\lambda_{1}=1$ & $\alpha_{1}=0.2$ & $I(0)=0.05$ & $\xi=0.0625$ \\
\hline$\lambda_{2}=0.1$ & $\alpha_{2}=0.02$ & $R(0)=0$ & $\delta=0.0525$ \\
\hline \multicolumn{4}{|c|}{$T=1000$} \\
\hline
\end{tabular}

According to (4) the reproduction number for this example equals to $R_{0}=1.1905$ and there exists an asymptotically stable endemic equilibrium point $(0.4640,0.4962,0.0009,0.0056)$ that system tends to this point.

By using constant parameter control $\mathrm{u}_{1}=0.3, \mathrm{u}_{2}=0, \mathrm{u}_{3}=0$ one can compute the basic reproductive number by (5) and obtain $R_{0}=0.8658$. The system tends to disease-free equilibrium point $(0.5,0.5,0,0)$ and outbreak does not occur. Here 


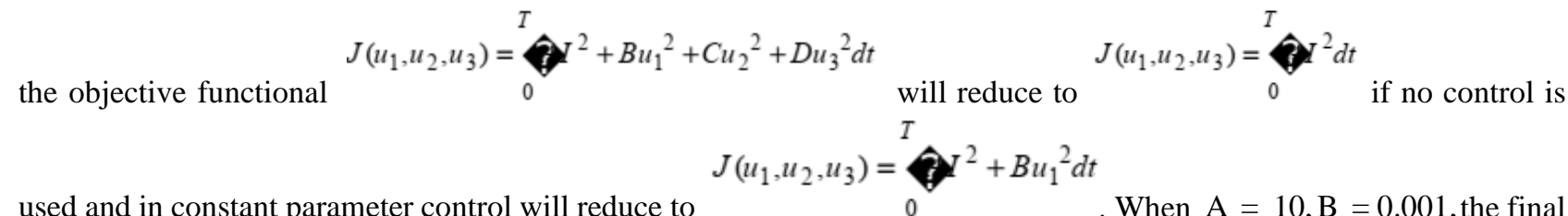
used and in constant parameter control will reduce to $\quad 0 \quad$. When $\mathrm{A}=10, \mathrm{~B}=0.001$, the final
cost is $\mathrm{J}=0.3117$ when we have no control on processes and $J=0.2199$ for constant parameter control strategy. Despite the control of outbreak, it seems that the costs are increase greatly. One can solve the simple optimization problem to find the best $\left(u_{1}, u_{2}, u_{3}\right)$. This gives $u_{1}=0.14, u_{2}=0.015, u_{3}=0.034, R_{0}=0.9662$ and $\mathrm{J}=0.1790$. Figure (2) shows the minimum cost static controls.
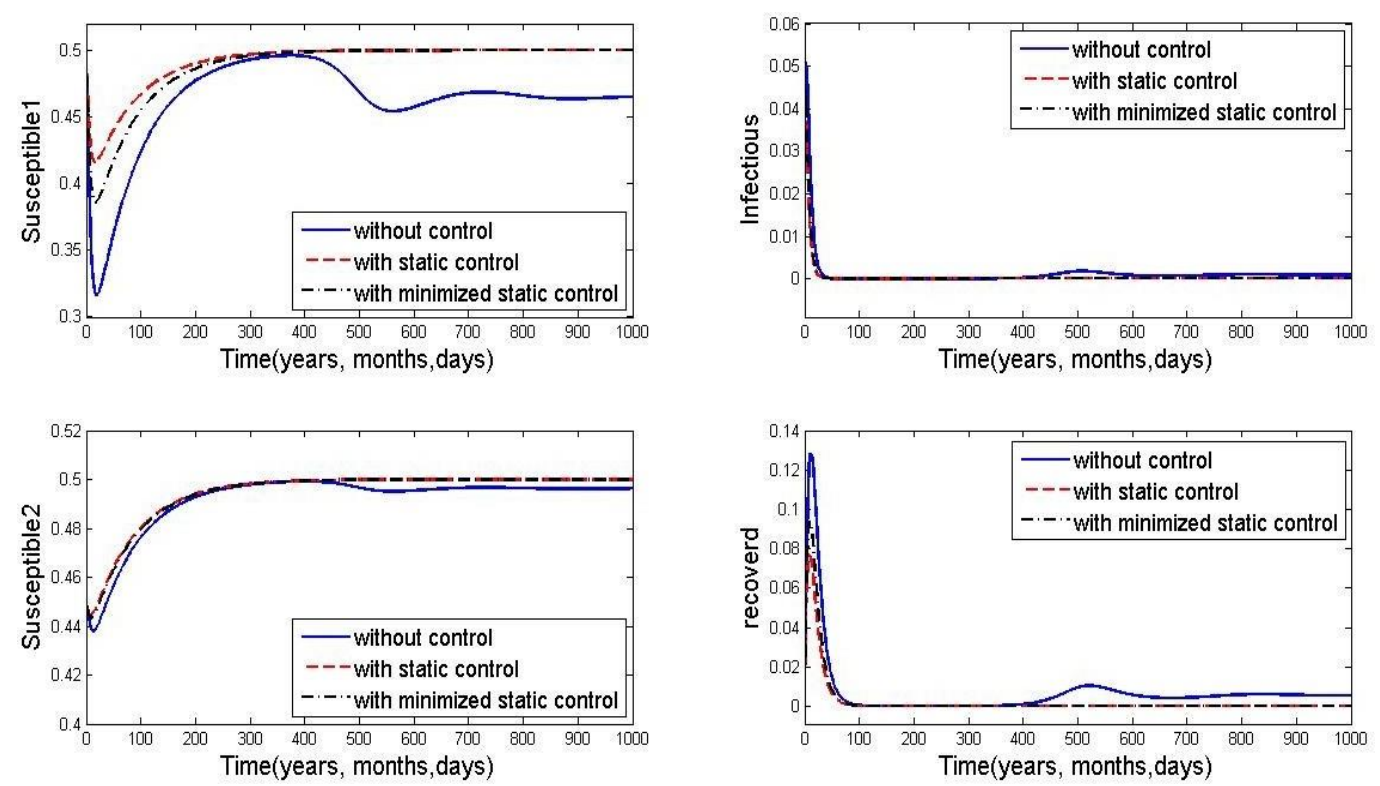

Figure 2: Minimum cost static control and it's effect on epidemic spreading. Using controlparameters $\left(u_{1}, u_{2}, u_{3}\right)$ causes the system to tend to disease-free equilibrium point.

\subsection{Example2}

Here, we consider the previous example with optimal control approach that control function ui can be continuous function with respect to the time. Let us enter the following values as an adjustment parameters inoptimal control method in the previous example:

\begin{tabular}{|c|c|}
\hline \multicolumn{2}{|c|}{ Parameters andvalues } \\
\hline $\mathrm{A}=10$ & $\mathrm{~B}=0.001$ \\
\hline $\mathrm{C}=0.001$ & $\mathrm{D}=0.01$ \\
\hline \multicolumn{2}{|c|}{$\mathrm{T}=1000$} \\
\hline
\end{tabular}

As we observe, the model system tends to endemic equilibrium. Figures (3) and (4) shows an optimal schedule and related control functions $\left(u_{1}, u_{2}, u^{3}\right)$ for $T=1000$. Final cost for optimal control is $J_{l}=0.0345$ and the final cost without control strategy is $J_{2}=0.3117$. The optimal control strategy tries to hold costs in a practical level and it depends on our adjusting objective functional $\mathrm{J}$ and coefficients $\mathrm{A}, \mathrm{B}, \mathrm{C}$ and $\mathrm{D}$.
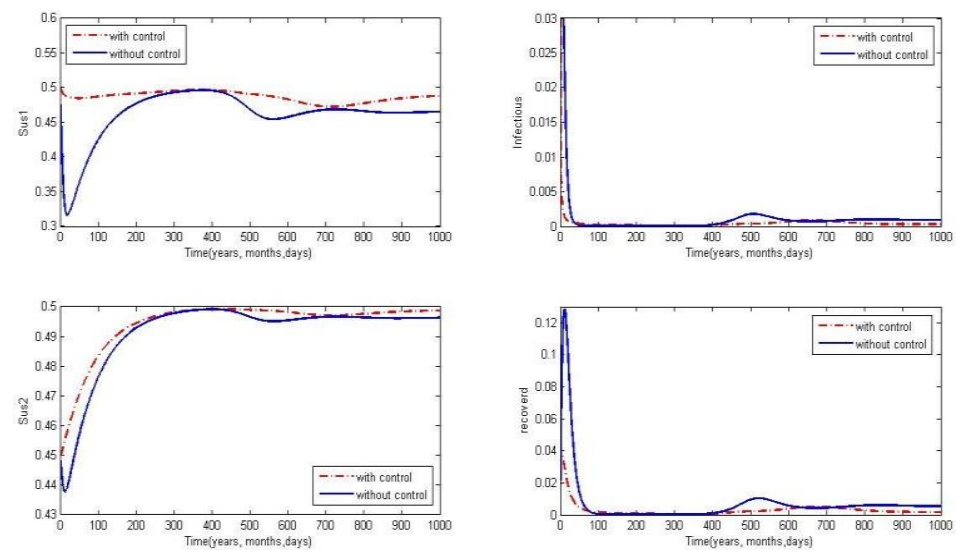

Figure 3: Optimal control strategy. obviously optimal control causes the model system not to tendto endemic equilibrium point and this process ocures with the minimum cost. 

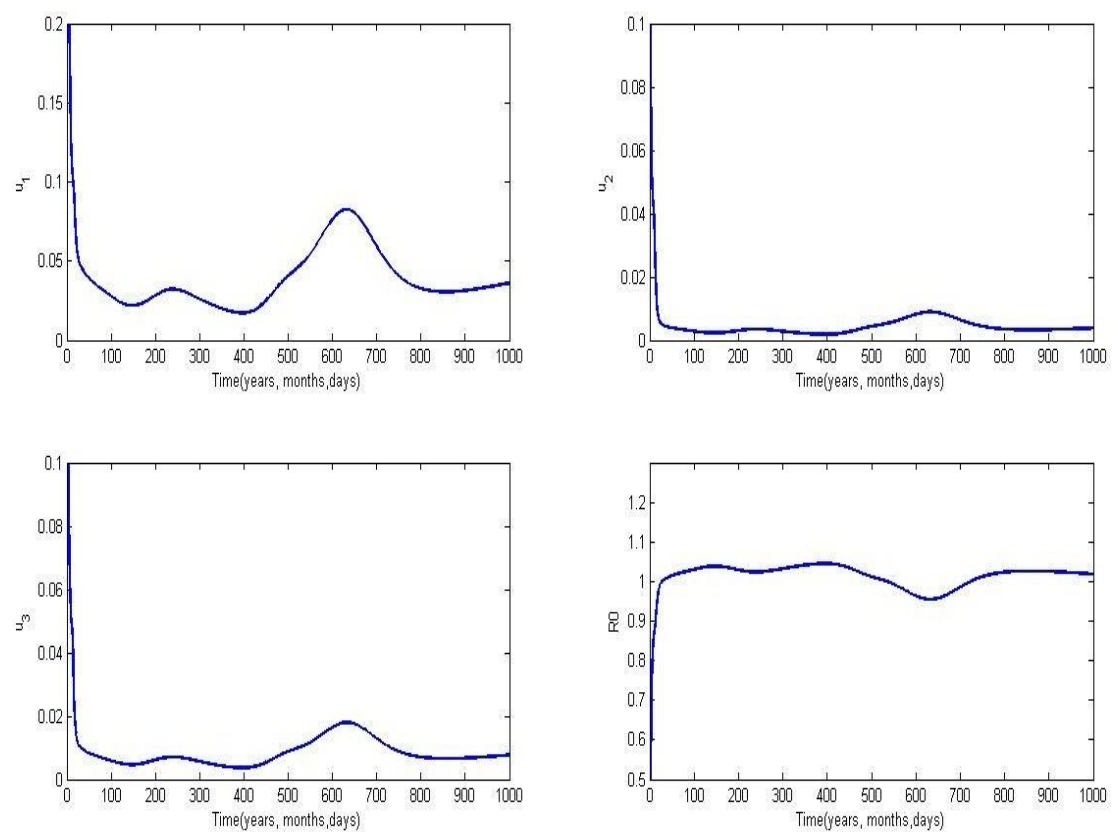

Figure 4: Control function. These functions show control startegy in every time. The last plot in thisfigure shows the change of basic reproduction ratio during the optimal strategy.

Let us change the objective functional (3) to

$$
J\left(u_{1}, u_{2}, u_{3}\right)=\stackrel{T}{T} \mathbf{I}+B u_{1}^{2}+C u_{2}^{2}+D u_{3}^{2} d t
$$
equations and control functions replace in the control system. Here, we want to reduce the deathstatistics. Let us enter the following values in the model system:

$A=10, B=0.001, C=0.001, D=0.01, \mathrm{~T}=350$. The final cost is $J=1.7131$ and when we have no control on processes and $\mathbf{J}=8.9168$ for optimal control strategy. In this manner, the control system approximate disease-free equilibrium point. The next figure shows the impact of these changes in evolution of epidemicspreading.
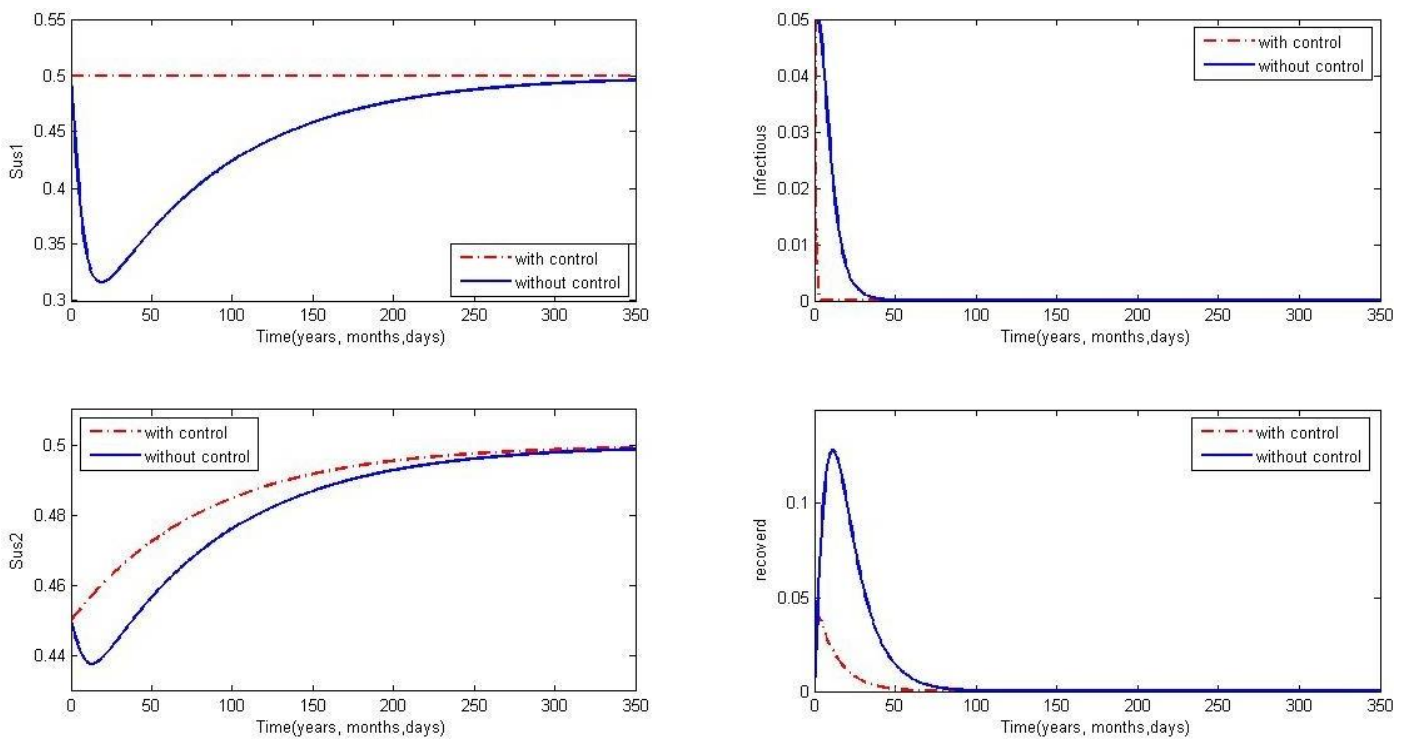

Figure 5: Optimal control causes the model system approximate to disease free equilibriumpoint and this process occurs with the minimum cost. 

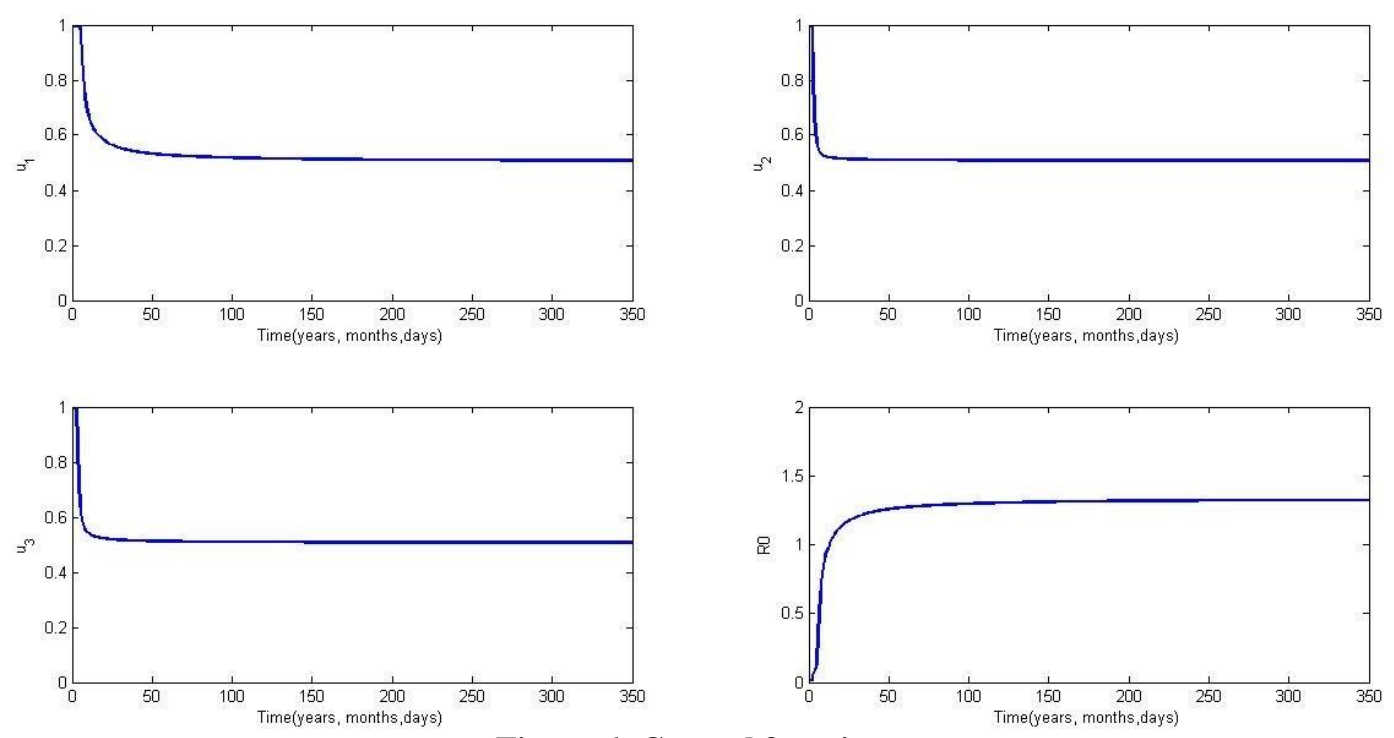

Figure 6: Control functions

\subsection{Example 3}

Let us neglect the partitioning susceptible into two groups and use simple SIR model only. Consequently, we have only two control functions

$\mathrm{u}_{\mathrm{p}}$ and $\mathrm{u}_{\mathrm{t}}$. These control functions have to be bounded on $[0,1]$. $\mathrm{u}_{\mathrm{p}}$ measures the time dependent efforts on the preventive strategy of susceptible individuals ins ${ }_{1}, \mathrm{~S}_{2}$, to reduce the number of individuals that may be infectious. The control function $u_{t}$ measure the time dependent efforts on the treatment of infected individuals in I. The next figures show optimal control and control functions in simple.
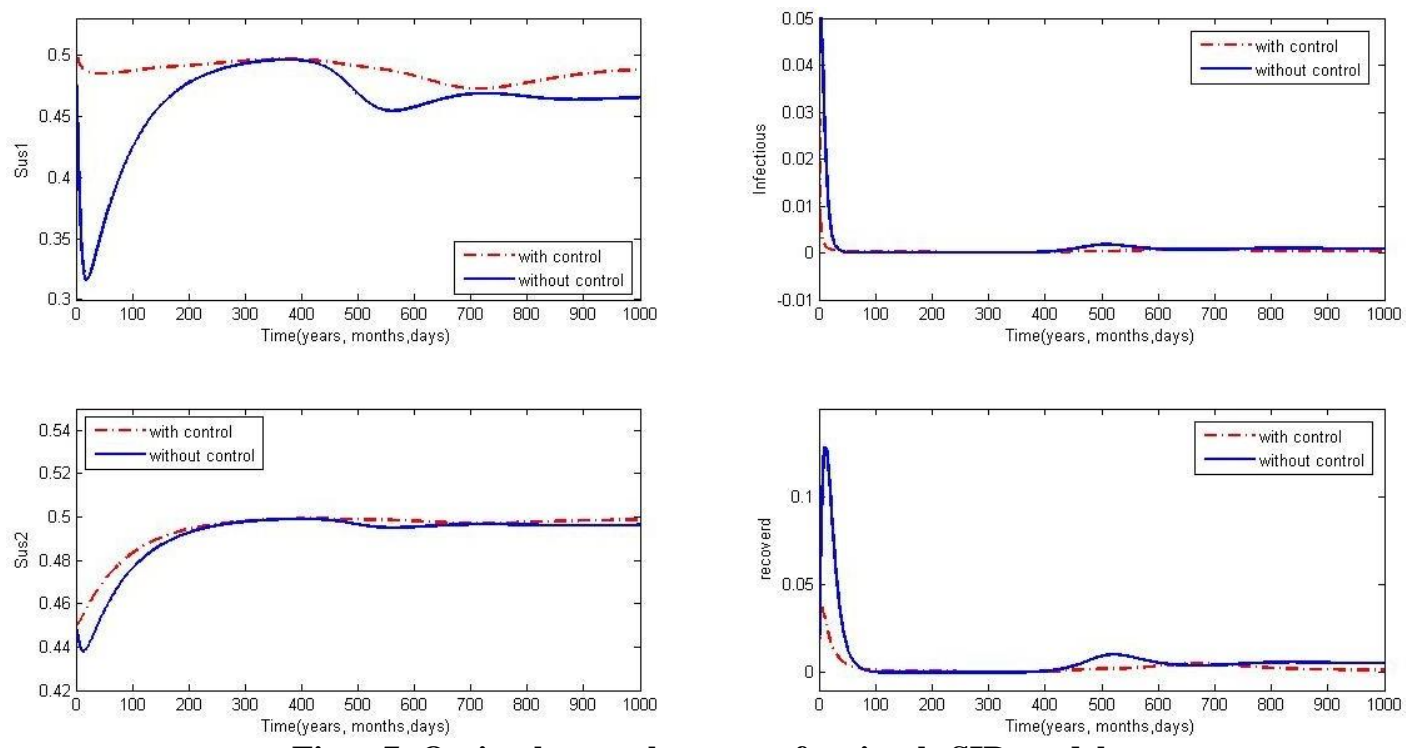

Figur 7: Optimal control strategy for simple SIR model. 

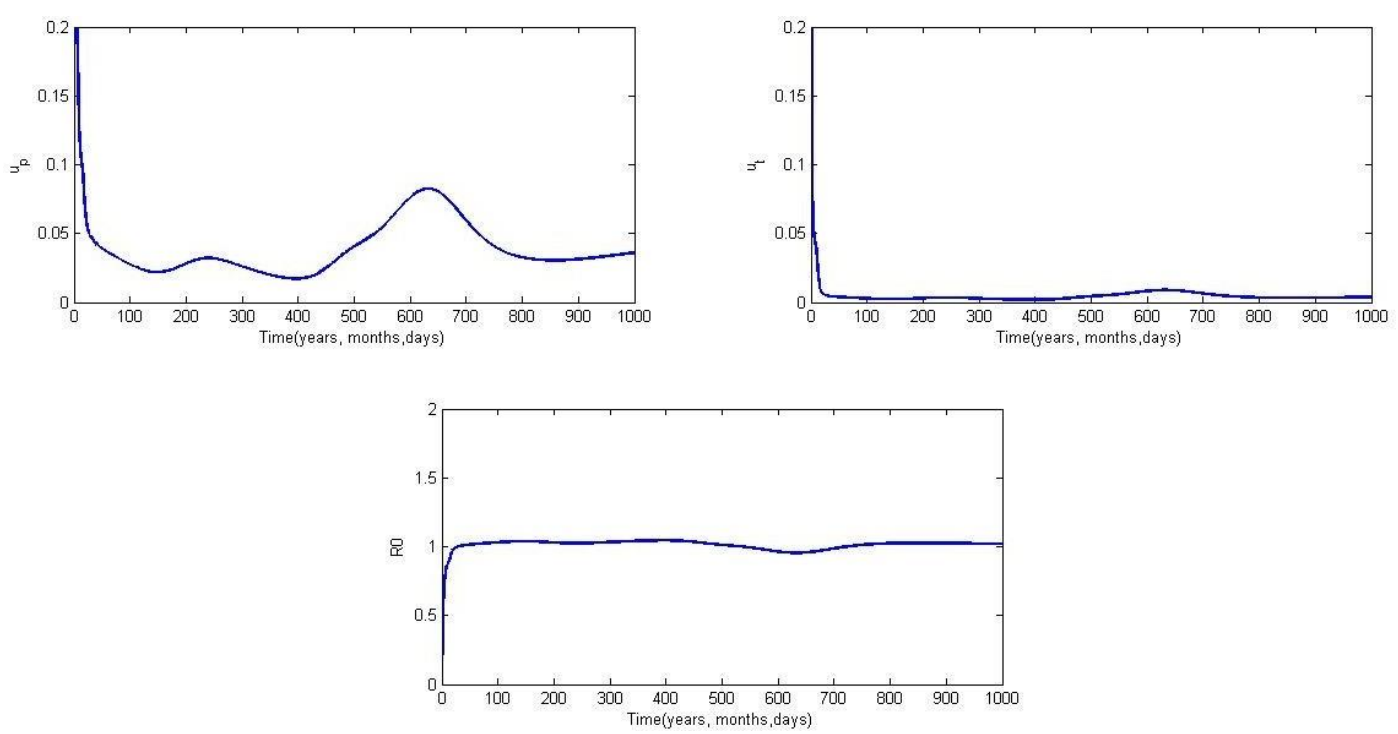

Figure 8: Control functions for simple SIR model.

Final cost for optimal control as we saw in example (2) is $\mathbf{J}=0.0345$ and the final cost without partitioning into two groups are $\mathrm{J}=0.0383$. These results show the efficiency of differential susceptibility optimal controlmethod.

\section{CONCLUSION}

This paper has presented mathematical and epidemiological results about the control of disease spreading inDS model. This was designed to examine the following questions:

- How does susceptible dividing into two groups can affect the dynamics of disease spreading?

- Which conditions on the rate of control parameter can ensure the eradication of disease, or at least minimizeits incidence

- What is the mathematical and numerical consequence of considering such groups?

- What is the optimal treatment and prevention strategy?

A deterministic mathematical model for the transmission dynamics of disease in DS model has been built to answer these questions. An important result of this analysis is that the cost-effective balance of prevention and treatment methods can control a disease outbreak. Strategies of optimal control can affect the reducing ofdeath toll and severity of an outbreak. Optimal control theory in our model is a starting point for more elaborate models.

\section{ACKNOWLEDGMENT}

This material is based upon work supported by the Islamic Azad University-ZanjanBranch.

\section{REFERENCES}

[1].Abiodun GJ, Marcus N, Okosun KO, Wittbooi P.J(2013), A model for control of HIV/AIDS with parental care, in: International Journal of Biomathematics, Volume 06, Issue 02.

[2].Bolker B.M, Grenfell B.T,(1993), Chaos and biological complexity in measles dynamics, Proc. R.Soc. Lond. B. 251, $75-81$.

[3].Capasso V,(1993), Mathematical structure of the epidemic systems, in: Lecture Notes in Biomathematics, vol. 97, Springer-Verlag, Berlin, Heidelberg.

[4].Castillo-Chavez C, Cooke K.L, Huang W, Levin S.A. (1989), On the role of long incubation periods in the dynamics of acquired immunodeficiency syndrome (AIDS). Part 2: Multiple group models. In: Castillo-Chavez, C. (ed.) Mathematical and Statistical Approaches to AIDS Epidemiology, (Lecture Notes in Biomathematics, Vol. 83) Springer-Verlag, Heidlberg.

[5].Cleary T.G(1988)., SCytoxin-producing Escherichia coli and the hemolytic uremic syndrome, Pediatric. Clinicsof North America 35 485-501.

[6].Deuflhard, P. and Bornemann, F,(2002), Scientific computing with ordinary differential equations, volume 42. Texts in Applied Mathematics, Springer, Berlin.

[7].Duncan C.J, Duncan S.R, Scott S,(1996), Whooping cough, epidemic in London 1701- 1812, infection dynamics, seasonal forcing and the effects of malnutrition. Proc. R. Soc. Lond. B. 263, 445-450 .

[8].Fister K, Donnelly J, (2005), Immunotherapy: An optimal control theory approach, Mathematical Biosciencesand Engineering, 499-510.

[9]. Fleming W. H, Rishel, R(1975), Deterministic and Stochastic Optimal Control, Springer Verlag, New York.

[10]. Folashade B.A, Marcus N, Okosun K.O.(2012) Application of optimal control to the epidemiology of malaria, Electronic Journal of Differential Equations, No. 81, pp. 1-22. 
[11]. Gaff H, Schaefer E.( 2009) Optimal control applied to vaccination and treatment strategies for various epidemiological models, Mathematical Biosciences and Engineering, 6.

[12]. Hethcote, H.W., York, J.A.(1984), Gonorrhea Transmission Dynamics and control, Lecture Notes inBiomathematics vol. 56, Springer-Verlag, Berlin, Heidelberg.

[13]. Horikoshi H, Kinomoto M, Sasao F, Mukai T, Luftig R.B, Ikuta K,(2001), Differential susceptibility of resting CD4+ T lymphocytes to a T-tropic and a macrophage (M)- tropic human immunodeficiency virus type1 is associated with their surface expression of CD38 molecules,Virus Research 73, 1-16.

[14]. Huang,W., Cooke, K.L., Castillo-Chavez (1992), Stability and bifurcation for a multiplegroup model for the dynamics of HIV/AIDS transmission, SIAM J. Appl. Math 52, 835-854.

[15]. Hyman J.M., Li J., Stanley E.A, (2001) The initialization and sensitivity of multigroup models for thetransmission of HIV, Journal of Theoretical Biology 208 227-249.

[16]. Hyman J.M, Li J(2005), Differential susceptibility epidemic models, Journal of Mathematical Biology 50 (6)626644.

[17]. Joshi H.R,(2002), Optimal control of an HIV immunology model, Optimal Control Applications and Methods, Volume 23, Issue 4, pages 199213, July/August.

[18]. Jung. E, lenhart S, Feng Z (2002), Optimal control of treatments in a two-starin tuberculosis model,Discrete and continuous dynamical systems-seriesB, Volume 2, Number 4, November

[19]. Kirschner D, Lenhart S, Serbin S (1997). Optimal control of the chemotherapy of HIV, Journal ofMathematical Biology, 35: 775-792.

[20]. Lenhart S, Workman, J.T (2007), Optimal Control Applied to Biological Models, Chapman Hall.LondonMa Z, Li J,(2009), Dynamical Modeling and Analysis of Epidemics, World Scientific, 2009.

[21]. Sethi, S. P. and G. L. Thompson,(2000) Optimal Control Theory: Applications to Management Science and Economics, Kluwer, Boston, 2nd edition.

[22]. Signore C, (2001), Rubeola, Primary Care Update for Ob/Gyns. 8, 138-140

[23]. Thieme H.R.(1984), Mathematics in Population Biology, Princeton University Press.

[24]. Waterai S, Yokota K, Tana, Kishimoto T, Kanadani T, Taketa K, Oguma K,(2001), Relationship between susceptibility to hemolytic-uremic syndrome and levels of globotriaosylceramide in human sera,Journal of Clinical Microbiology 39 798-800. 